\title{
Vía clínica para pacientes hospitalizados con fractura osteoporótica de cadera
}

\author{
Clinical pathway in hospitalized patients \\ with osteoporotic hip fracture
}

\author{
Rafael Monte-Secades ${ }^{1}$, Pablo Codesido-Vilar², Francisco Javier Pardo-Sobrino³, Emilia Irene García-Monasterio², \\ Amparo Portero-Vázquez ${ }^{4}$, Manuel Garcia-Novio ${ }^{5}$, Fernando Fernández-Lamelo ${ }^{6}$ \\ ${ }^{1}$ Servicio de Medicina Interna. ${ }^{2}$ Servicio de Cirugía Ortopédica y Traumatología. ${ }^{3}$ Servicio de Anestesiología y Reanimación. \\ ${ }^{4}$ Servicio de Rehabilitación. ${ }^{5}$ Servicio de Urgencias. ${ }^{6}$ Dirección de Procesos Asistenciales con Ingreso \\ Hospital Universitario Lucus Augusti. SERGAS. Lugo
}

\section{Introducción}

La fractura osteoporótica de cadera constituye uno de los problemas de salud con mayor repercusión sanitaria, no solo en cuanto a su impacto hospitalario sino también por sus consecuencias sobre la dependencia funcional de los enfermos y su supervivencia, a corto y largo plazo. El progresivo envejecimiento de la población predice un aumento progresivo de su prevalencia en los próximos años.

En España, en el año 2010, se registraron 45.210 fracturas primarias de cadera (76\% mujeres) y se ha estimado que en un plazo de 10 años desde 2012, se producirán 263.351 fracturas de cadera en mujeres y 84.213 en hombres. Aunque en los últimos años la incidencia ajustada por edad se ha estabilizado, existe una tendencia a aumentar en los grupos de mayor edad (>80 años). La mortalidad hospitalaria por fractura de cadera en nuestro país se sitúa en el 4,3\%, aumenta hasta el $5-10 \%$ al mes del alta hospitalaria y es del 30\% a los 12 meses. Por otra parte, se estima que el $30 \%$ de los enfermos que sobreviven, presentan una discapacidad significativa al año de la fractura y que solamente el 40-50\% recuperan la situación funcional que tenían antes de la fractura.

La población del área sanitaria de Lugo es una de las más envejecidas de España y por tanto presenta una elevada prevalencia de enfermedades crónicas, polimedicación y de osteoporosis. Estas circunstancias generan un elevado riesgo de complicaciones y dificultades de manejo clínico cuando estos enfermos ingresan por una fractura de cadera, de tal manera que se puede considerar esta patología como "una enfermedad médica ingresada en una planta quirúrgica". De ahí la necesidad de una colaboración estrecha y coordinada entre todos los servicios implicados en la asistencia de estos enfermos. En el Hospital Universitario Lucus Augusti (HULA) de Lugo se registran alrededor de 370 fracturas de cadera anuales, con tendencia a un incremento leve en los últimos 5 años. Desde hace más de 10 años existe un programa de atención preferente a los pacientes con fractura de cadera, desarrollado entre los servicios de Cirugía
Ortopédica y Traumatología y Medicina Interna. Fruto de esta colaboración y de las mejoras implantadas en la asistencia de estos enfermos, la mortalidad hospitalaria ha disminuido desde un 9,3\% en el año 2003 hasta un 2,6\% en el 2014, la estancia media ha descendido desde 17 días en el 2003 hasta 10 en el 2014 y la estancia prequirúrgica, desde 5,1 a 3,5 días. No obstante, existen amplias áreas de mejora de la asistencia, que atañen principalmente a la coordinación entre facultativos y de estos con el personal de enfermería, a la estandarización de procedimientos diagnósticos o terapéuticos y a la gestión del alta hospitalaria.

En este trabajo se propone una vía clínica para pacientes con fractura de cadera osteoporótica ingresados en el HULA, con los siguientes objetivos principales:

- Creación de un equipo multidisciplinar que implique a todos los "actores" involucrados en la asistencia de estos enfermos.

- Diseño de los flujos de trabajo implicados en el proceso asistencial.

- Actualización de todos los protocolos asistenciales de diagnóstico y tratamiento de acuerdo con la mejor evidencia científica disponible.

- Situar al paciente en el centro de todo el proceso asistencial.

\section{Vía Clínica. Metodología y objetivos}

Una vía clínica es un plan asistencial que se aplica a enfermos cuya patología tiene un curso clínico determinado y predecible, como ocurre en el caso de la fractura de cadera osteoporótica. Se caracteriza por la coordinación de una secuencia de procedimientos médicos, de enfermería, administrativos y de todo el personal sanitario implicado en su atención, con el fin de de lograr la mayor calidad asistencial y eficiencia en el proceso. Los protocolos y actuaciones previstas en la vía clínica sirven de guía a los profesionales, pero no están diseñados para reemplazar su juicio clínico. 
Los objetivos generales de una vía clínica son:

- Establecer una secuencia de actuaciones basadas en las mejores evidencias.

- Especificar la estructura y los niveles de coordinación entre profesionales.

- Conseguir el compromiso de la institución con el plan asistencial.

- Constituir una herramienta educativa para residentes.

- Servir de canal para la información al paciente y familiares durante el proceso.

- Constituir un marco para la investigación de la efectividad de las medidas adoptadas y tratamientos.

- Servir de herramienta para disminuir los efectos adversos asociados a la patología concreta y al proceso asistencial.

- Servir de marco para la medida de resultados de salud, de la calidad asistencial y de la eficiencia.

El grupo de trabajo que se ha responsabilizado de la realización de esta vía clínica ha estado integrado por representantes de los siguiente servicios: Cirugía Ortopédica y Traumatología (COT), Medicina Interna (MI), Anestesiología y Reanimación (AR), Rehabilitación (RHB), Enfermería y Dirección Médica. Entre los meses de enero y septiembre de 2015 se han celebrado varias reuniones de trabajo con el objeto de diseñar los flujos de trabajo y consensuar las actuaciones a considerar en la atención del enfermo que ingresa por fractura osteoporótica de cadera. Cada integrante del grupo ha elaborado los protocolos asistenciales que son competencia de su especialidad y área de conocimiento, los cuales se han revisado y validado en las reuniones generales del equipo. Para la confección de determinados planes de actuación se contó la colaboración de los servicios de Urgencias (URG) y Trabajo Social (TS). Las actuaciones y protocolos empleados, basados en el análisis de la mejor evidencia científica, se han adaptado a las características y dinámica de trabajo del hospital para el que se ha diseñado esta vía clínica (HULA), y por tanto sus conclusiones no deben generalizarse sin el preceptivo análisis en otros centros hospitalarios.

La bibliografía utilizada para la elaboración de esta vía clínica se detalla el final del documento. El grado de recomendación de las actuaciones se refiere a la fuerza de las evidencias que avalan dichas medidas, de acuerdo con las directrices marcadas por la SIGN (Scottish Intercollegiate Guidelines Network): A, basada en metaanálisis, revisión sistemática 0 ensayo clínico aleatorizado de calidad alta y muy bajo riesgo de presentar sesgos, directamente aplicables a la población diana; B, basada en estudios del nivel A extrapolados a la población diana, o bien en revisiones sistemáticas, estudios caso-control o de cohortes de alta calidad aplicables a la población diana, con muy bajo riesgo de presentar sesgos; C, basada en evidencias de nivel $\mathrm{B}$ extrapoladas a la población diana, o en estudios de caso-control o cohortes bien diseñados con bajo riesgo de presentar sesgos y una probabilidad moderada de demostrar una relación causal, directamente aplicables a la población diana; D, basada en evidencias de nivel $\mathrm{C}$ extrapolables a la población diana o bien en series de casos u opinión de expertos. Estos niveles de evidencia no reflejan la importancia clínica de la recomendación. Por otra parte, se definen las actuaciones de buena práctica clínica (ABP) como recomendaciones de práctica clínica basadas en la experiencia del grupo de trabajo de la vía clínica.

Los objetivos asistenciales generales planteados en esta vía clínica para pacientes con fractura osteoporótica de cadera son:

- Valoración precoz de comorbilidad.

- Conciliación de la medicación domiciliaria.

- Cirugía precoz, con preferencia en las primeras 24-48h posteriores al ingreso hospitalario.

- Establecimiento de protocolos asistenciales para los procedimientos y complicaciones más frecuentes: dolor, sonda vesical, manejo de perioperatorio de antigregantes y anticoagulantes, diabetes, profilaxis de enfermedad tromboembólica venosa (ETV), profilaxis antibiótica, transfusión de hemoderivados, síndrome confusional agudo, tratamiento de la osteoporosis.

- Asegurar un seguimiento clínico adecuado por parte del especialista en Traumatología y de Medicina Interna durante el ingreso.

- Proporcionar información al paciente y sus familiares por parte de todos los profesionales implicados en el proceso (principalmente COT y MI). La información ofrecida debe ser continuada y coherente entre los diferentes facultativos del equipo.

- Decisión consensuada e informe único de alta hospitalaria (COT y MI), en el que se reflejaran los comentarios y tratamientos indicados por ambos servicios cuando proceda.

- Todos los enfermos deben recibir tratamiento para la osteoporosis al alta hospitalaria.

\section{Equipo multidisciplinar. Plan general de trabajo}

La formación de un equipo multidisciplinar está basada en la coordinación de recursos sanitarios y del personal sanitario y no sanitario encargado de la atención, en este caso de los enfermos con fractura de cadera. Su funcionamiento estará guiado por el máximo respeto entre sus miembros y por el establecimiento de canales activos de comunicación en su seno, con énfasis en el contacto verbal.

Se programará una reunión semanal con los integrantes del equipo de multidisciplinar de trabajo, formado por al menos un profesional de los siguientes servicios y estamentos, en función de las necesidades asistenciales: COT, MI, AR, Enfermería, TS y RHB. En ella se programarán las actuaciones y procedimientos previstos para esa semana, incluyendo el plan de quirófano, incidencias y altas hospitalarias previstas. Todos los integrantes del equipo se podrán localizar mediante teléfono buscapersonas en horario ordinario de trabajo. 
A continuación se detalla el conjunto de actuaciones y protocolos de atención consensuados, mediante el análisis de los diferentes subprocesos que conforman el proceso asistencial de atención hospitalaria a la fractura de cadera del anciano. Entre paréntesis se señalan los servicios responsables de cada una de las actuaciones marcadas en el itinerario de atención.

\section{Subproceso en el área de Urgencias hospitalarias (URG y COT)}

Engloba desde la llegada del paciente al servicio de URG con fractura de cadera hasta su paso a la planta de hospitalización o sala quirúrgica

\section{Recepción y diagnóstico (URG)}

- El triaje y la valoración inicial incluirán una historia clínica en la que se determinará el grado de movilidad previo a la fractura y la medicación domiciliaria (D).

- Se administrará analgesia en el momento de la presunción diagnóstica, utilizando siempre la vía parenteral (D).

- Se solicitarán radiografía axial y anteroposterior de ambas caderas, electrocardiograma, hemograma, bioquímica con glucosa, iones, urea, creatinina y coagulación (D).

\section{Tratamiento (URG, COT) e ingreso (COT)}

- La fractura de cadera puede asociarse a hematomas graves, por lo que debe considerarse la administración de sueros para asegurar un estado óptimo de hidratación y volemia, teniendo en cuenta las características basales del enfermo (edad, cardiopatia, neumopatía, etc). No existen preferencias para el uso de ningún tipo de solución en particular (D).

- Se aplicará el protocolo analgésico establecido para la fractura de cadera (tabla 1). No se considera indicado el empleo rutinario de la tracción preoperatoria, ya sea cutánea 0 esquelética $(\mathbf{A})$.

- Se tendrá en cuenta la conciliación de la medicación domiciliaria, de acuerdo con estos principios generales: suspender los fármacos innecesarios o redundantes; ajustar la necesidad de fármacos a la situación clínica de ingreso (ej no pautar antihipertensivos si coexiste hipovolemia); no introducir benzodiacepinas, AINES ni anticolinérgicos (ABP).
- Se informará al paciente y/o familiares (COT) sobre el diagnóstico y tratamiento recomendado. Se procederá a solicitar consentimientos para cirugía y transfusión de hemoderivados en los casos que proceda (ABP).

- Se cursarán interconsultas preferentes a los servicios de Ml y AR (en el caso de que no se disponga de un programa de asistencia compartida), así como una petición analítica ordinaria que incluya hemograma, iones, función renal, calcio, fósforo, proteínas totales, albúmina $y$ vitamina $D(A B P)$.

\section{Subproceso de hospitalización prequirúrgica (Enfermería, COT, AR, MI)}

Engloba la recepción e ingreso del paciente en la sala de hospitalización para la aplicación de las medidas de tratamiento adecuadas para la fractura de cadera, hasta el traslado al bloque quirúrgico o el alta hospitalaria

\section{Recepción en planta de hospitalización (Enfermería)}

- Se realizará una valoración enfermera al ingreso según las necesidades y principios de Virginia Henderson. Se aplicarán de forma estandarizada las escalas de Braden de prevención de úlceras por presión y la escala visual analógica para valorar la intensidad del dolor (EVA) (D).

- Se formulará un plan estandarizado de cuidados (anexo 1), de acuerdo con los diagnósticos que entran dentro de la competencia del profesional de enfermería y que éste puede tratar de forma independiente (NANDA -North American Nursing Diagnosis Association-), los resultados que dependen de las acciones enfermeras y que son clínicamente útiles (NOC -Nursing Outcome Classification-) y las intervenciones enfermeras que pueden favorecer dichos resultados (NIC -Nursing Intervention Classification-) (ABP).

- Se adoptarán las siguientes medidas, de aplicación estandarizada a todos los pacientes: suplementos nutritivos hiperproteicos por vía oral (C); oxígeno suplementario (2 litros por minuto por gafas nasales) si sat02<93\% (monitorización mediante pulsioximetría diaria) (B); evitar estreñimiento (lactulosa 15 mg/12h si >48h sin deposición, enema de limpieza si >72h sin deposición) (ABP).

Tabla 1. Analgesia en la fractura de cadera (D)

\begin{tabular}{|l|}
\hline PRINCIPIOS GENERALES \\
\hline EI dolor relacionado con la cirugía de la fractura de cadera se considera de intensidad moderada (European Task Force Management, 1998) \\
\hline La analgesia debe ser pautada en las ordenes de tratamiento, nunca se administrará "si dolor" \\
\hline $\begin{array}{l}\text { La vía analgésica de elección será la intravenosa. Se sustituirá por analgesia oral con buen } \\
\text { control analgésico y a partir de la tolerancia oral de los mismos }\end{array}$ \\
\hline Es recomendable pautar analgesia de rescate, protección gástrica y rescate antiemético \\
\hline PAUTA ANALGÉSICA \\
\hline Paracetamol 1-2 gr/6-8h iv a todos los pacientes salvo contraindicación médica \\
\hline Rescate: metamizol magnésico 2gr/8h iv \\
\hline $\begin{array}{l}\text { En caso de mal control de dolor o dolor postoperatorio: morfina 1-2 amp + primperán } 2 \text { amp en } 250 \mathrm{ml} \text { de suero salino en } \\
\text { perfusión ev, con inicio en 5-10 ml/h. Individualizar dosis y ritmo en función de la edad y comorbilidad delpaciente }\end{array}$ \\
\hline Se desaconseja el empleo rutinario de ketorolaco o dexketoprofeno \\
\hline
\end{tabular}


- La valoración enfermera inicial se completará con una ponderación de las necesidades socio-sanitarias del paciente y el contacto directo con la unidad de Trabajo Social si fuera preciso, al comienzo del ingreso (ABP).

Valoración de comorbilidad y terapeútica (MI, COT)

La valoración por parte del especialista en medicina interna se realizará el primer día de estancia en la planta de hospitalización. Su papel debe centrarse en la identificación de los problemas médicos principales del enfermo incluyendo su nivel de dependencia funcional (índice de Barthel, anexo 2), la valoración del riesgo de complicaciones que estos condicionan en relación a la intervención planteada y en la indicación de las medidas terapéuticas necesarias para optimizar el estado del enfermo en ese contexto. Cualquier decisión de solicitar nuevas pruebas diagnosticas o de pautar tratamientos preoperatorios que demoren la cirugía debe ponderarse cuidadosamente con los riesgos que supondría el retraso de la cirugía. El internista debe responsabilizarse de realizar un seguimiento clínico adecuado y continuado del paciente desde el ingreso hasta el fin de la hospitalización (B).

\section{Protocolos asistenciales por síntomas y patologías específicas (Ml, AR)}

Se han consensuado protocolos de atención clínica estandarizados para algunas de las patologías y complicaciones más frecuentes durante el ingreso por fractura osteoporótica de cadera, así como para el manejo de determinados fármacos: dolor (tabla 1), diabetes mellitus (tabla 2), síndrome confusional agudo (tabla 3), antiagregación (tabla 4), anticoagulación y terapia puente (tablas 5 y 6), profilaxis antibiótica (tabla 7), profilaxis de enfermedad tromboembólica venosa (ETV) (tabla 8), transfusión de hemoderivados (tabla 9) y osteoporosis (tabla 10).

Valoración de riesgo anestésico (AR)

El especialista de AR valorará el riesgo anéstesico (estratificación ASA -American Society of Anesthesiologists-) y solicitará el consentimiento informado para la realización del procedimiento anestésico. Se considera como técnica anestésica de elección el bloqueo neuraxial (epidural o espinal), puesto que se asocia a una menor mortalidad temprana postoperatoria y a una menor incidencia de complicaciones postquirúrgicas (A), especialmente en cuanto al desarrollo de síndrome confusional. En enfermos que reciban tratamiento con antiagregantes (clopidogrel, doble antiagregación) y presenten indicación de cirugía urgente, se valorará la realización de anestesia general.

Se consideran causas clínico-analíticas de aplazamiento de la intervención quirúrgica y que precisan valoración médica previa a la cirugía (AR, MI), las siguientes (D):

- Hemoglobina $<8 \mathrm{gr} / \mathrm{dl}$.

- $\mathrm{Na}<1200>150 \mathrm{mEq} / \mathrm{l}$.

- Coagulopatía reversible: INR debe ser <2 para una intervención quirúrgica con anestesia general y debe ser $<1,5$ para intervención quirúrgica con bloqueo neuraxial (anestesia raquídea).

- Glucemia $>400$ mg/dl.

- Creatinina $>2,6 \mathrm{mg} / \mathrm{dl}$.

- Sepsis.

- Arritmia con Fc>120 Ipm.

Tabla 2. Manejo perioperatorio de diabetes mellitus (D)

\section{PRINCIPIOS GENERALES}

El tratamiento hospitalario se basará en la modificación de la pauta en función

de la monitorización de la glucemia y la optimización de ajustes en función de la situación clínica.

Todos los pacientes deben tener una determinación de hemoglobina glucosilada si no se ha realizado en los 3 meses previos al ingreso.

Se aconsejan unos objetivos de glucemia preprandial menor de $130 \mathrm{mg} / \mathrm{dl} \mathrm{y} \mathrm{posprandial} \mathrm{menor} \mathrm{de} \mathrm{180-200} \mathrm{mg/dl.}$

En general el tratamiento de la hiperglucemia durante la hospitalización se realizará con insulina.

La planificación del tratamiento al alta dependerá del grado de control metabólico

previo a la hospitalización y de la evolución durante el ingreso.

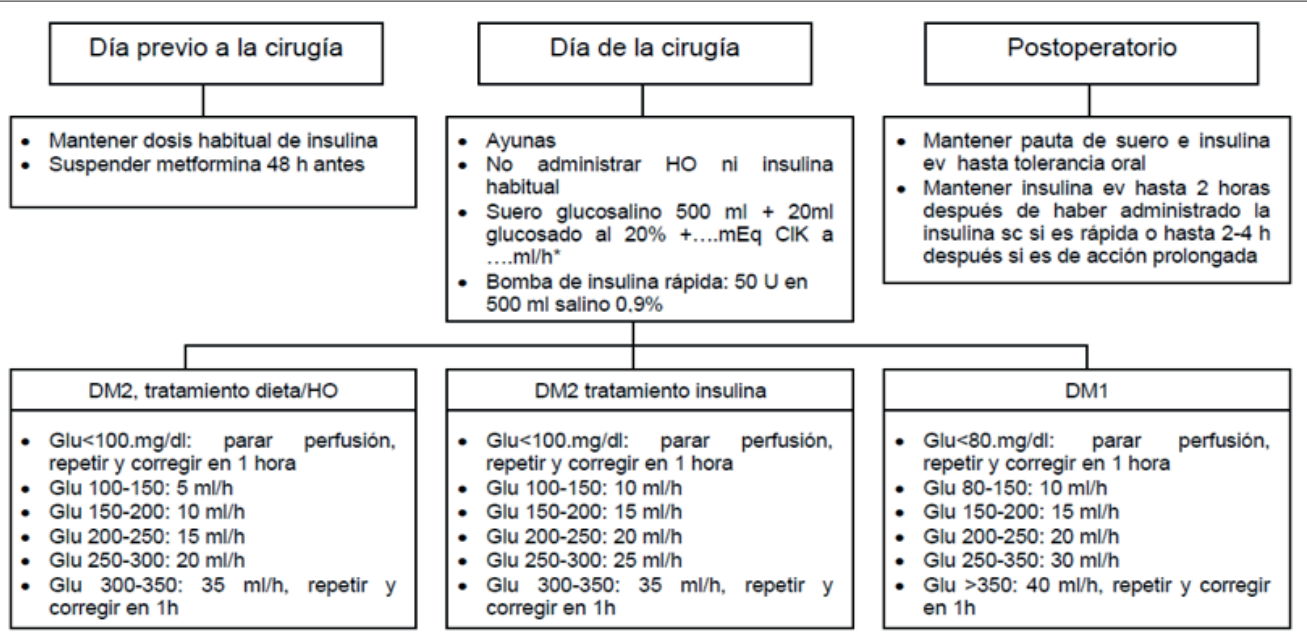

HO: hipoglucemiantes orales

*Ajustar ritmo de perfusión y mEq CIK según situación clínica y comorbilidad basal 


\section{Subproceso de tratamiento quirúrgico (COT)}

Engloba la anestesia, la intervención quirúrgica y la reversión anestésica de una fractura de cadera en el anciano.

El objetivo asistencial en todos los enfermos en los que se considere indicado el tratamiento quirúrgico será realizar la intervención en las primeras 24 (preferente) 048 horas de ingreso hospitalario (C). Demorar más tiempo la intervención en pacientes estables aumenta la mortalidad, las complicaciones y la estancia hospitalaria. En pacientes inestables se considerará un plazo inferior a 72 horas. En el caso de considerar necesaria una intervención quirúrgica urgente, ésta será indicada por los servicios de COT y AR.

Se valorará la adopción de un tratamiento conservador (no quirúrgico) en las siguientes situaciones: estado clínico que no tolera una intervención quirúrgica, pacientes con una esperanza de vida limitada a días en la que la cirugía no aporta beneficio, en enfermos con situación basal de dependencia funcional e incapacidad para la deambulación que presenten una fractura de cadera sin dolor (consensuado por el equipo) y en los que no desean ser operados (ABP).

El tipo de intervención quirúrgica a considerar dependerá del tipo de fractura, de la presencia de enfermedad articular previa y de las condiciones basales generales del paciente:

\section{Fractura intracapsular (cabeza y cuello de fémur)}

- Enfermedad articular degenerativa previa, vida activa y expectativa de vida razonable: artroplastia total (B).

- No enfermedad articular degenerativa previa:.

- Fractura no desplazada (Garden I-fractura impactaday II-fractura completa sin desplazamiento-): osteosíntesis (tornillos canulados) (A)

- Fractura desplazada (Garden III-fractura completa con desplazamiento parcial- y IV-fractura completa con desplazamiento total-) (B)

$\sim>75$ años: en casos de vida activa se considerará la osteosíntesis (tornillos canulados). En caso de mala calidad de vida previa es preferible la artroplastia parcial.

>75 años: artroplastia parcial

\section{Fractura extracapsular (no existen diferencias significativas entre las diferentes técnicas) (A)}

- Fractura per/intertrocantérea.

- Estables (clasificación OTA/AO): se considerará preferentemente el dispositivo intramedular (enclavado gamma); en pacientes con vida activa y sin comorbilidad se valorará la colocación de un dispositivo extramedular (dinamic hip screw).

- Inestables (clasificación OTA/A0): dispositivo intramedular (enclavado gamma)

- Fractura subtrocantérea (se consideran todas inestables): dispositivo intramedular (enclavado gamma).
Tabla 3. Prevención y tratamiento del síndrome confusional agudo (B, ABP)

\begin{tabular}{|c|c|}
\hline PREVENCIÓN & TRATAMIENTO (OPCIONES) \\
\hline $\begin{array}{l}\text { Evitar aislamiento sensorial: } \\
\text { asistencia de familiares, } \\
\text { gafas, audífono. }\end{array}$ & $\begin{array}{l}\text { Tratar enfermedades } \\
\text { desencadenantes. }\end{array}$ \\
\hline $\begin{array}{l}\text { Separar pacientes con delirium } \\
\text { si comparten habitación. }\end{array}$ & $\begin{array}{l}\text { Haloperidol } \\
\text { 0,5-1mg/6h vo (1 gota-0,1 mg) } \\
2,5 \mathrm{mg} \mathrm{im} \mathrm{(1} \mathrm{amp-5} \mathrm{mg)}\end{array}$ \\
\hline $\begin{array}{l}\text { Minimizar el uso de medidas } \\
\text { de restricción física. }\end{array}$ & $\begin{array}{l}\text { Tiaprida } \\
\text { 100-200 mg/12h vo } \\
\text { (1 comp-100 mg) } \\
100 \text { mg ev (1 amp-100 mg) }\end{array}$ \\
\hline Movilización precoz. & $\begin{array}{l}\text { Risperidona } \\
\text { 0,25-0,5 mg/12h vo } \\
\text { (1 comp/ml-1mg) }\end{array}$ \\
\hline Tratamiento adecuado del dolor. & $\begin{array}{l}\text { Olanzapina } \\
\text { 2,5 mg/24h (comp de } \\
2,5,5 \text { y } 10 \mathrm{mg})\end{array}$ \\
\hline Corregir estreñimiento. & $\begin{array}{l}\text { Considerar suplementos de } \\
\text { tiamina en todos los casos. }\end{array}$ \\
\hline Minimizar el uso de sonda vesical. & $\begin{array}{l}\text { Evitar benzodiazepinas salvo } \\
\text { en casos especiales. }\end{array}$ \\
\hline Eliminar fármacos innecesarios. & \\
\hline Oxigenoterapia. & \\
\hline \multicolumn{2}{|c|}{$\begin{array}{l}\text { Fármacos asociados a delirium: } \\
\text { - Narcóticos: meperidina, morfina } \\
\text { - AlNES } \\
\text { - Benzodiacepinas } \\
\text { - Inhibidores de colinesterasa: donezepilo } \\
\text { - Ranitidina, Antieméticos } \\
\text { - Dopaminérgicos: levodopa, bromocriptina } \\
\text { - Antidepresivos: mirtazapina, IRS*, tricíclicos } \\
\text { - Anticomiciales: fenitoina, valproato, levetiracetam } \\
\text { - Otros: propranolol, digoxina, diuréticos, hipoglucemiantes }\end{array}$} \\
\hline \multicolumn{2}{|c|}{ Se desaconseja el uso de fármacos (ej. neurolépticos) como profilaxis. } \\
\hline
\end{tabular}

Tabla 4. Manejo perioperatorio de la antiagregación (D, ABP)

\section{AAS}

AAS y AINEs no necesitan intervalo de seguridad

(no administrar el día de la cirugía)

Dosis altas de AAS (ej. $300 \mathrm{mg}$ ), se recomienda

sustituir al ingreso por AAS $100 \mathrm{mg} / \mathrm{d}$

TRIFUSAL, CILOSTAZOL

No necesitan intervalo de seguridad (no administrar el día de la cirugía)

CLOPIDOGREL, PRASUGREL, TICAGRELOR

Interrupción 7 días antes de una anestesia neuraxial

Valorar dosis de carga de clopidogrel al reiniciarlo tras

$>5 \mathrm{~d}$ de suspensión en caso de stent farmacoactivo

No realizar terapia puente para la intervención

quirúrgica con otro antiagregante

\section{DOBLE ANTIAGREGACIÓN}

Riesgo trombótico con la interrupción bajo o moderado (Stent coronario convencional, cirugía revascularización coronaria, IAM, ACV/AIT,

IPC>6semanas; Stent farmacoliberador >6meses): mantener el AAS y suspender el otro antiagregante (habitualmente clopidogrel o ticagrelor)

Riesgo trombótico con la interrupción alto (resto de supuestos):

individualizar y tomar una decisión multidisciplinar

*En ausencia de sangrado activo, se considerará la reintroducción del tratamiento

antiagregante a las 24 horas de la intervención quirúrgica. En casos de elevado riesgo

de traombosis se puede valorar su reinicio a las 6 horas de finalizada la cirugía. 


\section{Subproceso de hospitalización postquirúrgica} (COT, MI, RHB, Enfermería)

Engloba el cuidado y tratamiento del paciente desde su traslado procedente de la Unidad de Reanimación, tras la intervención quirúrgica, hasta su alta hospitalaria.

\section{Protocolos asistenciales de actuación}

- Los cuidados enfermeros se aplicarán según el plan estandarizado NANDA-NIC-NOC especificado (anexo 1). Se mantiene activos también en este subproceso los protocolos de atención clínica para el manejo del dolor (tabla 1), diabetes mellitus (tabla 2), síndrome confusional agudo (tabla 3), antiagregación (tabla 4), anticoagulación y terapia puente (tablas 5 y 6), profilaxis de enfermedad tromboembólica venosa (ETV) (tabla 8), transfusión de hemoderivados (tabla 9) y osteoporosis (tabla 10), al igual que las medidas de aplicación estandarizada respecto a la administración de suplementos hiperproteicos y al control del estreñimiento.

- Se administrará oxigenoterapia (gafas nasales 2 lpm) durante al menos 72 horas después de la intervención quirúrgica, o más tiempo si se detecta hipoxemia (monitorización mediante pulsioximetría). Su empleo puede reducir la frecuencia de eventos cardiovasculares (B).

- No se recomienda el sondaje vesical periquirúrgico rutinario (B). Sus indicaciones son: retención aguda de orina, incontinencia urinaria que comprometa la herida quirúrgica y la monitorización de la diuresis. Si la sonda vesical se ha colocado en el quirófano, se retirará a las 24 horas de la cirugía. Se valorará la opción de realizar cateterismo vesical intermitente regular (ej cada 8h) durante 48 horas (ABP).

- Se retirarán los drenajes quirúrgicos de la herida cuando estos sean escasamente productivos, preferentemente en las primeras 24 horas desde su colocación (A).

Rehabilitación funcional (RHB, COT)

Todos los pacientes con fractura de cadera son susceptibles de recibir una atención de rehabilitación multidisciplinar con la intención de recuperar su grado de independencia funcional previo a la fractura (B). El objetivo es conseguir que el paciente intervenido de su fractura de cadera, sea capaz de caminar de forma autónoma antes del alta hospitalaria. Se realizará una interconsulta preferente al servicio de RHB a

Tabla 5. Tiempos mínimos de suspensión de anticoagulantes antes de la intervención quirúrgica y reinicio posterior (B)

\begin{tabular}{|l|l|l|}
\hline & SUSPENDER & REINICIAR $^{\star *}$ \\
\hline Acenocumarol & Hasta objetivos INR ${ }^{\star}$ & $48-72 \mathrm{~h}$ \\
\hline Dabigatran & $72 \mathrm{~h}\left(\mathrm{FG}{ }^{\dagger}<5096 \mathrm{~h}\right)$ & $48-72 \mathrm{~h}$ \\
\hline Rivaroxaban/Apixaban & $48 \mathrm{~h}(\mathrm{FG}<5072 \mathrm{~h})$ & $48-72 \mathrm{~h}$ \\
\hline HNF§ & $4-6 \mathrm{~h}$ & $12 \mathrm{~h}$ \\
\hline HBPM dosis profiláctica & $12 \mathrm{~h}$ & $12 \mathrm{~h}$ \\
\hline HBPM dosis anticoagulante & $24 \mathrm{~h}(12 \mathrm{~h} \mathrm{si} \mathrm{TEP}<1 \mathrm{~m})$ & $48-72 \mathrm{~h}$ \\
\hline Fondaparinux & $36-48 \mathrm{~h}$ & $12 \mathrm{~h}$ \\
\hline
\end{tabular}

${ }^{*}$ INR permitidos para intervención quirúrgica: bloqueo neuraxial $<1,5$; anestesia general $<2$. Corregir con vitamina $\mathrm{K}$ o plasma fresco en caso de cirugía urgente.

${ }^{*}$ No reanudar hasta conseguir una hemostasia adecuada

FG: filtrado glomerular

sHNF: heparina no fraccionada

¿HBPM: heparina de bajo peso molecular

Tabla 6. Terapia puente anticoagulante (la fractura de cadera se considera de riesgo hemorrágico alto -categoría cirugía mayor $>45$ min-) (D, ABP)

\begin{tabular}{|c|c|c|c|}
\hline & ANTIVITAMINA K & HBPM & $\begin{array}{l}\text { ANTICOAGULANTES } \\
\text { DE ACCIÓN DIRECTA }\end{array}$ \\
\hline $\begin{array}{l}\text { Riesgo } \\
\text { trombótico alto* }\end{array}$ & $\begin{array}{l}\text { 1. Suspender antivitK y administrar } 1 \text { amp de vit K } \\
\text { ev si se programa intervención en plazo de } 24 \mathrm{~h} \text {. } \\
\text { 2. Pedir coagulación la mañana siguiente. } \\
\text { 3. Pautar HBPM (enoxaparina } 1 \mathrm{mg} / \mathrm{Kg} / 12 \mathrm{~h} \text { ). } \\
\text { Inicio con INR<2. } \\
\text { 4. Tiempos de suspensión y reinicio } \\
\text { de acuerdo con la tabla } 5 \text {. } \\
\text { 5. A las } 12 \mathrm{~h} \text { de la intervención } \\
\text { pautar dosis de HBPM profiláctica. }\end{array}$ & $\begin{array}{l}\text { 1. Cambiar a HBPM en } 2 \text { dosis } \\
\text { (enoxaparina } 1 \mathrm{mg} / \mathrm{kg} / 12 \mathrm{~h} \text { ). } \\
\text { 2. Tiempos de suspensión y reinicio } \\
\text { de acuerdo con la tabla } 5 \text {. }\end{array}$ & $\begin{array}{l}\text { 1. Decisión multidisciplinar. } \\
\text { 2. Pauta de profilaxis ETV con } \\
\text { HBPM\&. Inicio al término de efecto } \\
\text { de la dosis del anticoagulante. } \\
\text { 3. Tiempos de suspensión y reinicio } \\
\text { de acuerdo con la tabla } 5 \text {. }\end{array}$ \\
\hline $\begin{array}{l}\text { Riesgo } \\
\text { trombótico } \\
\text { moderado } \\
\text { o bajo }\end{array}$ & $\begin{array}{l}\text { 1. Suspender anticoagulación. } \\
\text { 2. Pauta de profilaxis ETV con } \\
\text { HBPM. Iniciar con INR<2. } \\
\text { 3. Tiempos de suspensión y reinicio } \\
\text { de acuerdo con la tabla } 5 \text {. }\end{array}$ & $\begin{array}{l}\text { 1. Suspender anticoagulación. } \\
\text { 2. Pauta de profilaxis ETV con } \\
\text { HBPM. Inicio según vida media } \\
\text { de la HBPM suspendida. } \\
\text { 3. Tiempos de suspensión y reinicio } \\
\text { de acuerdo con la tabla } 5 \text {. }\end{array}$ & $\begin{array}{l}\text { 1. Suspender anticoagulación. } \\
\text { 2. Pauta de profilaxis ETV con HBPM. } \\
\text { Iniciar a las } 24 \text { de la suspensión. } \\
\text { 3. Tiempos de suspensión y reinicio } \\
\text { de acuerdo con la tabla } 5 \text {. }\end{array}$ \\
\hline
\end{tabular}

*Riesgo trombótico alto: válvula cardiaca metálica, ACV/AIT<6 meses, FA CHADS2 25 , válvula reumática mitral, ETV $<3$ meses 0 trombofilia grave. Riesgo trombótico moderado o bajo: resto de supuestos

\&El efecto predecible de los anticoagulantes de acción directa tras su suspensión, no hace necesaria la terapia puente anticoagulante 
las 24 horas de la intervención quirúrgica, quien programará las actividades de rehabilitación funcional individualizadas para cada paciente, contando con la presencia de un fisioterapeuta adscrito a la planta de hospitalización de COT (ABP). La movilización precoz postquirúrgica mejora la capacidad de recuperación funcional. En ausencia de complicaciones y bajo la supervisión del servicio de COT, se promoverá la sedestación inmediata en dispositivos estables, la bipedestación a las 24-48 horas y la deambulación con ayuda a las 48 horas de la intervención quirúrgica (ABP).

\section{Alta hospitalaria y transición a domicilio}

(COT, MI, Enfermería, RHB)

El informe clínico de alta hospitalaria debe ser un documento único que incluya las actuaciones realizadas durante el ingreso, las complicaciones desarrolladas durante el mismo, Ios nuevos diagnósticos realizados y el tratamiento ambulatorio recomendado (ABP). Para ello, el alta se programará cuando sea factible con al menos 24 horas de antelación y se incluirá un addendum del servicio de Ml en el informe cuando proceda, con identificación del internista consultor. Se adjuntará asimismo un informe de cuidados de enfermería de acuerdo con el plan estandarizado NIC.

Se especificará en todos los casos que proceda el diagnóstico de fractura osteoporótica en el informe de alta hospitalaria y todos los pacientes recibirán al alta tratamiento contra la osteoporosis según el protocolo asistencial establecido (tabla 10) (ABP). Por otra parte, se determinarán las necesidades de analgésicos, se continuará con las medidas de profilaxis para la ETV (tabla 8) y se considerará la indicación de suplementos de hierro, ácido fólico y vitamina B12. Todas estas medidas deben conciliarse con la medicación domiciliaria del paciente (ABP)

Se protocolizarán revisiones ambulatorias en el informe de alta por parte de los servicios de COT y Atención Primaria. En función de las necesidades de cada paciente y del criterio clínico, se efectuarán revisiones en los servicios de Ml y RHB (ABP).

Tabla 7. Profilaxis antibiótica $(\mathbf{A})$

CEFAZOLINA 1gr (<80kg) $02 \mathrm{gr}(>80 \mathrm{~kg})$

30-60 minutos antes de la incisión quirúrgica, y cada $6 \mathrm{~h} 3$ dosis postquirúrgicas (total 24h) independientemente del uso de redones.

ALÉRGICOS A PENICILINA: Teicoplanina $400 \mathrm{mg}$ + gentamicina 240 mg*

30-60 minutos antes de la incisión quirúrgica, dosis única, independientemente del uso de redones.

*Adaptado a las características microbiológicas de las infecciones protésicas del HULA (Lugo)

Tabla 8. Profilaxis de ETV (A)

\begin{tabular}{|l|}
\hline $\begin{array}{l}\text { HPM en dosis de alto riesgo de ETV (opciones) } \\
\cdot \\
\cdot \text { Enoxaparina } 40 \mathrm{mg} \mathrm{sc} / 24 \mathrm{~h}\end{array}$ \\
\hline Iniciar el día del ingreso salvo intervención quirúrgica urgente. \\
\hline Tiempos de suspensión y reinicio de acuerdo con la tabla 5. \\
\hline Continuar hasta 4 semanas después del alta hospitalaria. \\
\hline En caso de trombopenia por heparina: fondaparinux 2,5 mg sc/24h. \\
\hline
\end{tabular}

Tabla 9. Transfusión de hemoderivados (B)

\section{CONCENTRADO DE HEMATÍES}

Anemia aguda perioperatoria, transfundir (2 unidades) si

- $\mathrm{Hb}<7 \mathrm{gr} / \mathrm{dl}$ en paciente menor de 65 años, previamente sano

- $\mathrm{Hb}<8 \mathrm{gr} / \mathrm{dl}$ en paciente mayor de 65 años

$\mathrm{Hb}<9 \mathrm{gr} / \mathrm{dl}$ en paciente con antecedentes de insuficiencia cardiaca o coronaria

Anemia crónica (orientativo)

$<5 \mathrm{gr} / \mathrm{dl}$, transfundir

5 - $9 \mathrm{gr} / \mathrm{dl}$, según criterio clínico

- $10 \mathrm{gr} / \mathrm{dl}$ no transfundir

\section{PLAQUETAS}

Profiláctica (1 pool): si plaq<50.000/mm³ prequirófano

Terapéutica (1 pool): hemorragia atribuible a defecto plaquetario cualitativo 0 plaq $<50.000 \mathrm{~mm}^{3}$

\section{PLASMA FRESCO CONGELADO}

Profiláctico (10 ml/kg): pacientes en tratamiento con antivitamina $\mathrm{K}$ o con déficit de factores vitamina $\mathrm{K}$ dependientes, que precisen cirugía urgente Terapéutico (10 ml/kg): hemorragia grave atribuible a déficit de factores vitamina $\mathrm{K}$ dependientes 
Tabla 10. Valoración y Tratamiento de la osteoporosis (ABP)

\section{DIAGNÓSTICO}

Bioquímica: hemograma, coagulación, función renal, calcio, fósforo, proteínas totales, y vitamina D. Resto de paeticiones según criterio clínico

Radiografía de cadera y columna dorsolumbar

\section{TRATAMIENTO}

Dieta rica en lácteos (3 vasos de leche/d + 1 yogurt aportan $1000 \mathrm{mg}$ calcio) con preferencia sobre suplementos de calcio

Vitamina D

- Si insuficiencia de vitD (20-29 ng/ml): calcifediol 266 mcg 1 cada 7 días 6 semanas y después 1 quincenal

- Si deficiencia de vitD (<20 ng/ml): calcifediol 266 mcg 1 cada 48h 6 semanas y después 1 quincenal

\section{TRATAMIENTO ADICIONAL A LA VITAMINA D}

Se considerará tratamiento con antirresortivos u otros en aquellos enfermos con un pronóstico vital estimado por su situación basal superior a 2 años

Bifosfonatos (preferente)

- No iniciar bifosfonatos durante el ingreso, diferir hasta 4 semanas después del alta

- Paciente con FG > 35 y buena adherencia a tto oral y tolerancia digestiva: bifosfonato oral (alendronato 75mg/semana o risedronato $35 \mathrm{mg} / \mathrm{semana}$ )

- Paciente con FG > 35 y mala adherencia a tto oral o intolerancia digestiva: bifosfonato ev (zoledronato), pautar 6 semanas después del alta. En estos casos es conveniente disponer de una determinación adicional de vit $\mathrm{D}$ (solo si se parte de déficit grave) y calcio antes de la administración

Denosumab (60 mg sc/6 m): considerar en pacientes con FG < 35. Iniciar 4 semanas después del alta

Teriparatida (20 mcg sc/d): considerar en pacientes con fractura atípica o fallo de tratamiento con antirresortivos

Anexo 1. Plan estandarizado de cuidados enfermeros NANDA-NOC-NIC (ABP)

\begin{tabular}{|c|c|c|c|c|c|}
\hline \multicolumn{2}{|c|}{ DIAGNÓSTICOS NANDA } & \multicolumn{2}{|c|}{ NOC (Resultados) } & \multicolumn{2}{|c|}{ NIC (Intervención) } \\
\hline 00085 & $\begin{array}{l}\text { Deterioro de la } \\
\text { movilidad física }\end{array}$ & & Movilidad & $\begin{array}{l}0140 \\
1806 \\
1840 \\
1801 \\
3540 \\
5602 \\
5820 \\
430 \\
5612\end{array}$ & $\begin{array}{l}\text { Fomentar la mecánica corporal } \\
\text { Ayuda con el autocuidado: transferencia. } \\
\text { Ayuda con el autocuidado: micción/defecación. } \\
\text { Ayuda con el autocuidado: baño/higiene } \\
\text { Prevención de úlceras por presión } \\
\text { Enseñanza: Proceso de enfermedad. } \\
\text { Disminución de la ansiedad } \\
\text { Control intestinal. } \\
\text { Enseñanza actividad/ejercicio prescrito }\end{array}$ \\
\hline 00035 & Riesgo de lesión & $\begin{array}{l}1828 \\
0916\end{array}$ & $\begin{array}{l}\text { Conocimiento: Prevención de caídas. } \\
\text { Nivel de delirio }\end{array}$ & $\begin{array}{l}6490 \\
6440\end{array}$ & $\begin{array}{l}\text { Prevención de caídas } \\
\text { Manejo del delirio }\end{array}$ \\
\hline 00004 & Riesgo de infección & 0204 & $\begin{array}{l}\text { Consecuencias de la } \\
\text { inmovilidad: fisiológicas }\end{array}$ & $\begin{array}{l}0704 \\
2930 \\
2300 \\
6680 \\
1870 \\
1876 \\
2314 \\
4238 \\
3320 \\
\end{array}$ & $\begin{array}{l}\text { Cuidados del paciente encamado. } \\
\text { Preparación quirúrgica } \\
\text { Administración de medicación } \\
\text { Monitorización de los signos vitales } \\
\text { Cuidados del drenaje. } \\
\text { Cuidados del catéter urinario } \\
\text { Administración de medicación: intravenosa (iv) } \\
\text { Flebotomía: muestra de sangre venosa. } \\
\text { Oxigenoterapia. }\end{array}$ \\
\hline 00046 & $\begin{array}{l}\text { Deterioro de la } \\
\text { integridad cutánea }\end{array}$ & 1102 & $\begin{array}{l}\text { Curación de la herida: } \\
\text { Por primera intención. }\end{array}$ & $\begin{array}{l}3660 \\
590 \\
620 \\
1100 \\
2080 \\
2210 \\
4030\end{array}$ & $\begin{array}{l}\text { Cuidado de las heridas. } \\
\text { Manejo de la eliminación urinaria. } \\
\text { Cuidados de la retención urinaria. } \\
\text { Manejo de la nutrición } \\
\text { Manejo de líquidos/electrólitos } \\
\text { Administración de analgésicos. } \\
\text { Administración de hemoderivados. }\end{array}$ \\
\hline
\end{tabular}


Anexo 2. Índice de Barthel: valoración del nivel de independencia del paciente con respecto a la realización de las actividades básicas de la vida diaria

\begin{tabular}{|c|c|c|}
\hline \multicolumn{3}{|l|}{ ÍNDICE DE BARTHEL } \\
\hline \multirow[t]{3}{*}{ Alimentación } & Independiente. Capaz de comer por sí solo en un tiempo razonable. La comida puede ser cocinada y servida por otra persona. & 10 \\
\hline & Necesita ayuda para cortar la carne, extender la mantequilla... pero es capaz de comer sólo. & 5 \\
\hline & Dependiente. Necesita ser alimentado por otra persona. & 0 \\
\hline \multirow[t]{2}{*}{ Lavado (baño) } & Independiente. Capaz de lavarse entero, de entrar y salir del baño sin ayuda y de hacerlo sin que una persona supervise. & 5 \\
\hline & Dependiente. Necesita algún tipo de ayuda o supervisión. & 0 \\
\hline \multirow[t]{3}{*}{ Vestido } & Independiente. Capaz de ponerse y quitarse la ropa sin ayuda. & 10 \\
\hline & Necesita ayuda. Realiza sin ayuda más de la mitad de estas tareas en un tiempo razonable. & 5 \\
\hline & Dependiente. Necesita ayuda para las mismas. & 0 \\
\hline \multirow[t]{2}{*}{ Arreglo } & $\begin{array}{l}\text { Independiente. Realiza todas las actividades personales sin ayuda alguna, } \\
\text { los complementos necesarios pueden ser provistos por alguna persona. }\end{array}$ & 5 \\
\hline & Dependiente. Necesita alguna ayuda. & 0 \\
\hline \multirow[t]{3}{*}{ Deposición } & Continente. No presenta episodios de incontinencia. & 10 \\
\hline & Accidente ocasional. Menos de una vez por semana o necesita ayuda para colocar enemas o supositorios. & 5 \\
\hline & Incontinente. Más de un episodio semanal. & 0 \\
\hline \multirow[t]{3}{*}{ Micción } & Continente. No presenta episodios. Capaz de utilizar cualquier dispositivo por si solo (botella, sonda, orinal,...). & 10 \\
\hline & $\begin{array}{l}\text { Accidente ocasional. Presenta un máximo de un episodio en } 24 \text { horas } \\
\text { o requiere ayuda para la manipulación de sondas } 0 \text { de otros dispositivos. }\end{array}$ & 5 \\
\hline & Incontinente. Más de un episodio en 24 horas. & 0 \\
\hline \multirow[t]{3}{*}{ Ir al retrete } & Independiente. Entra y sale solo y no necesita ayuda alguna por parte de otra persona. & 10 \\
\hline & Necesita ayuda. Capaz de manejarse con una pequeña ayuda; es capaz de usar el cuarto de baño. Puede limpiarse solo. & 5 \\
\hline & Dependiente. Incapaz de acceder a él o de utilizarlo sin ayuda mayor. & 0 \\
\hline \multirow[t]{4}{*}{ Traslado (cama/sillón) } & Independiente. No requiere ayuda para sentarse o levantarse de una silla ni para entrar o salir de la cama. & 15 \\
\hline & Mínima ayuda. Incluye una supervisión o una pequeña ayuda física. & 10 \\
\hline & Gran ayuda. Precisa ayuda de una persona fuerte o entrenada. & 5 \\
\hline & Dependiente. Necesita una grúa o el alzamiento por dos personas. Es incapaz de permanecer sentado. & 0 \\
\hline \multirow[t]{4}{*}{ Deambulación } & $\begin{array}{l}\text { Independiente. Puede andar } 50 \text { metros o su equivalente en casa sin ayuda supervisión. Puede utilizar } \\
\text { cualquier ayuda mecánica excepto un andador. Si utiliza una prótesis, puede ponérsela y quitársela solo. }\end{array}$ & 15 \\
\hline & Necesita ayuda. Necesita supervisión o una pequeña ayuda física por parte de otra persona o utiliza andador. & 10 \\
\hline & Independiente en silla de ruedas. No requiere ayuda ni supervisión. & 5 \\
\hline & Dependiente. Incluye ser rodado por otro. & 0 \\
\hline \multirow[t]{3}{*}{ Escaleras } & Independiente. Capaz de subir y bajar un piso sin ayuda ni supervisión de otra persona. & 10 \\
\hline & Necesita ayuda. Necesita ayuda o supervisión. & 5 \\
\hline & Dependiente. Es incapaz de salvar escalones. & 0 \\
\hline
\end{tabular}

La incapacidad funcional se valora como:

- Total: 0-20 puntos

- Grave: 21-60 puntos

- Moderada: 61-90 puntos
- Ligera: 91-99 puntos

- Independencia: 100 puntos
Puntuación Total:

Anexo 3. Pruebas complementarias diagnósticas protocolizadas, a realizar en todos los pacientes (ABP)

\begin{tabular}{|l|c|c|c|c|c|}
\hline & Área de Urgencias & $\begin{array}{c}\text { Ingreso en sala de } \\
\text { COT }\end{array}$ & $\begin{array}{c}\text { Postoperatorio } \\
\text { inmediato }\end{array}$ & Postoperatorio 24h & Postoperatorio 72h \\
\hline Hemograma & $X$ & & $X$ & $X$ & $X$ \\
\hline Coagulación & $X$ & & $X$ & $X$ & $X$ \\
\hline $\begin{array}{l}\text { Glucosa, iones, función } \\
\text { renal }\end{array}$ & $X$ & $X$ & & & \\
\hline $\begin{array}{l}\text { Ca, P, proténas totales, } \\
\text { albúmina, vitamina D }\end{array}$ & & & & & \\
\hline Radiografía tórax & $X$ & & $X$ & & \\
\hline Radiografía cadera & $X$ & & & & \\
\hline
\end{tabular}

* Según criterio clínico

Resto de pruebas según criterio clínico. 


\section{Bibliografía}

1. Avenell A, Handoll HH. Nutritional supplementation for hip fracture after care in older people. Cochrane Database Syst Rev. 2010 Jan 20; (1):CD001880

2. Bardales Mas Y, González Montalvo Jl, Abizanda Soler P, Alarcón Alarcón MT. Hip fracture guidelines. A comparison of the main recommendations. Rev Esp Geriatr Gerontol. 2012; 47: 220-7

3. Fractura de cadera en el anciano: proceso asistencial integrado. Sevilla. Consejería de Salud (2002). ISBN 84-8486-039-6. Andalucía. Consejería de Salud

4. Francis J, Aminoff MJ, Schmader KE, Wilterdink JL. Delirium and acute confusional states: Prevention, treatment, and prognosis. Disponible en www.uptodate.com (septiembre 2015)

5. Griffiths R, Alper J, Beckingsale A, Goldhill D, Heyburn G, Holloway J, Leaper E, Parker M, Ridgway S, White S, Wiese M, Wilson I. Management of proximal femoral fractures 2011: Association of Anaesthetists of Great Britain and Ireland. Anaesthesia. 2012; 67: 85-98

6. Handoll HH, Queally JM, Parker MJ. Pre-operative traction for hip fractures in adults Cochrane Database Syst Rev. 2011 Dec 7; (12): CD000168

7. Heidbuchel H, Verhamme P, Alings M, Antz M, Diener HC, Hacke W, Odgren J, Sinnaeve P, Camm AJ, Kirchhof P. Updated European heart rhythm association practical guide on the use of non-viamin $\mathrm{K}$ antagonist anticoagulants in patients with non-valvular atrial fibrillation. Europace. 2015; 17:1467-507

8. Hidalgo F, Gomez-Luque A, Ferrandis R, et al. Manejo perioperatorio de los anticoagulantes orales directos en cirugia urgente y sangrado. Monitorización y tratamiento hemostático. Rev Esp Anestesiol Reanim. 2015; 62: 450-60

9. Mak JC, Cameron ID, March LM; National Health and Medical Research Council. Evidence-based guidelines for the management of hip fractures in older persons: an update. Med J Aust. 2010; 192: 37-41

10. Bulecheck GM, Butcher HK, Dochterman JM. Clasificación de intervenciones de enfermería (NIC). Elsevier, 5 ed. 2009 (Barcelona)

11. Moorhead, S, Johnson M, Maas M. Clasificación de resultados de enfermería (NOC) Elsevier, 4 ed. 2009 (Barcelona)

12. Morrison RS, Siu AL, Schmader KE, Sokol HN. Medical consultation for patients with hip fracture. Disponible en http://www.uptodate.com (septiembre 2015)

13. Opperer M, Danninger T, Stundner O, Memtsoudis SG. Perioperative outcomes and type of anesthesia in hip surgical patients: An evidence based review. World J Orthop. 2014; 5: 336-43

14. Ortiz P, Mingo A, Lozano M, Vesga MA, Grifols JR, Castrillo A, Algora M, Romón I, Cárdenas JM; Sociedad Española de Transfusión Sanguínea. Guía sobre la transfusión de componentes sanguíneos. Med Clin (Barc) 2005; 125: 389-96
15. Parker MJ, Gurusamy K. Internal fixation versus arthroplasty for intracapsular proximal femoral fractures in adults. Cochrane Database Syst Rev. 2006 Oct 18; (4): CD001708

16. Parker MJ, Handoll HH. Gamma and other cephalocondylic intramedullary nails versus extramedullary implants for extracapsular hip fractures in adults. Cochrane Database Syst Rev. 2010 Sep 8; (9): CD000093

17. Pérez Pérez A, Conthe Gutiérrez P, Aguilar Diosdado M, Bertomeu Martínez V, Galdos Anuncibay P, García de Casasola G, Gomis de Bárbara R, Palma Gamiz JL, Puig Domingo M, Sánchez Rodríguez A. Tratamiento de la hiperglucemia en el hospital. Med Clin (Barc) 2009 4; 132: 465-75

18. Perez-Verdún MA, Sánchez-Cantalejo Ramírez A, Tirado Reyes M. Indicadores de calidad en un proceso integrado: fractura de cadera del anciano. Rehabilitación (Madrid) 2012; 46: 287-294

19. Ortiz P, Mingo A, Lozano M, Vesga MA, Grifols JR, Castrillo A, Algora M, Romón I, Cárdenas JM; Sociedad Española de Transfusión Sanguínea. Guía sobre la transfusión de componentes sanguíneos. Med Clin (Barc) 2005; 125: 389-96

20. Scotish intercollegiate guidelines network. Management of hip fracture in older people. A national clinical guideline. June 2009. Disponible en http://www.sign.ac.uk/ pdf/sign111.pdf (septiembre 2015)

21. Simunovic N, Devereaux PJ, Sprague S, Gordon H. Guyatt M, Schemitsch E, DeBee $J$, Bhandari M. Effect of early surgery after hip fracture on mortality and complications: systematic review and meta-analysis. CMAJ 2010; 182: 1609-1615

22. Sociedad Española de Cirugía Ortopédica y traumatología. Grupo de estudio e investigación de la Osteoporosis y la fractura osteoporótica (SECOT-GEIOS). Mesa M Caeiro JR, Calvo E, Carpintero P, Etxebarría I, Fgigueroa J, et al. Tratamiento multidiciplinar de la fractura de cadera. Vías clínicas de la fractura de cadera. Julio 2011. Disponible en https://www.secot.es/uploads/descargas/grupos trabajo/geios/ GEIOS_20100322082321_LIBRO_Tratamiento_multidisciplinar_de_la_fractura_de_femur.pdf (septiembre 2015)

23. Sociedad Española de Geriatría y Gerontología, Sociedad Española de Cirugía Ortopédica y Traumatología. Guía de buena práctica clínica en Geriatría. Anciano afecto de fractura de cadera 2007. Disponible en http://www.segg.es/sites/default/files/ page/guia_fractura_cadera.pdf (septiembre 2015)

24. Spyropoulos AC, Douketis JD. How I treat anticoagulated patients undergoimg an elective procedure or surgery. Blood 2012; 120: 2954-2962

25. Walker KM, Eiff P, Grayzel J. Hip fractures in adults. Disponible en www.uptodate. com (septiembre 2015) 